\title{
Error Bounds in the Rayleigh-Ritz Approximation of Eigenvectors
}

\author{
H. F. Weinberger
}

(June 9, 1960)

\begin{abstract}
The difference between any eigenvector $u_{p}$ of a linear operator $A$ and its Rayleigh-Ritz approximation $w_{p}$ is bounded in terms of the differences between the eigenvalues $\lambda_{i}$ of $A$ and their Rayleigh-Ritz upper bounds $\kappa_{i}$. The bound for the difference between $u_{p}$ and $w_{p}$ approaches zero with $\kappa_{p}-\lambda_{p}$.
\end{abstract}

\section{Introduction}

The most common method of approximating the eigenvalues $\lambda_{1} \leq \lambda_{2} \leq$. . of a symmetric linear operator $\mathrm{A}$ is the Rayleigh-Ritz method $[1,5,19] .{ }^{1}$ 'This reduces an eigenvalue problem on a space of a large or even infinite number of dimensions to an eigenvalue problem on a space of relatively few dimensions.

If the desired eigenvalues $\lambda_{i}$ are characterized as minima, the Rayleigh-Rifz approximations $\kappa_{\imath}$ give upper bounds for them.

Along with the upper bounds $\kappa_{i}$ for the eigenvalues the Rayleigh-Ritz method yields associated vectors $w_{i}$. It is to be expected that these vectors approximate the eigenvectors of $\mathrm{A}$ in some sense. Furthermore, it is to be expected that the better the eigenvalue $\lambda_{i}$ is approximated by $\kappa_{i}$, the better will be the approximation of $w_{i}$ to the corresponding eigenvector.

Indeed, this is easily seen in the case of the first eigenvector. If the unit vector $w_{1}$ is expanded in terms of the normalized eigenvectors $u_{i}$ of $\mathrm{A}$, we have

$$
\begin{aligned}
w_{1} & =\sum a_{i} u_{i}, \\
\sum a_{i}^{2} & =1,
\end{aligned}
$$

and

$$
\sum \lambda_{i} a_{i}^{2}=\kappa_{1}
$$

Subtracting $\lambda_{1}$ times (1.2) from (1.3) and noting that $\lambda_{2} \leq \lambda_{3} \leq$. . we find that

or equivalently

$$
a_{1}^{2} \geq \frac{\lambda_{2}-\kappa_{1}}{\lambda_{2}-\lambda_{1}}
$$

$$
\left[1-\frac{1}{2}\left(w_{1}-u_{1}, w_{1}-u_{1}\right)\right]^{2} \geq 1-\frac{\kappa_{1}-\lambda_{1}}{\lambda_{2}-\lambda_{1}}
$$

In general the $\lambda_{\imath}$ are unknown, and we must express

\footnotetext{
${ }_{1}^{1}$ Figures in brackets indicate the literature references at the end of this paper.
}

our results in terms of the $\kappa_{i}$ and any lower bounds $\bar{\lambda}_{i}$ for the $\lambda_{i}$ that may be available. Such bounds can be obtained by various methods (see for example $[1,2,3,4,5,6,10,11,12,13,14,19,20,21,22,23,24,25,26])$.

If $\lambda_{i} \geq \bar{\lambda}_{i}$, the inequality (1.5) leads to

$$
\left[1-\frac{1}{2}\left(w_{1}-u_{1}, w_{1}-u_{1}\right)\right]^{2} \geq 1-\frac{\kappa_{1}-\bar{\lambda}_{1}}{\overline{\lambda_{2}}-\overline{\lambda_{1}}}
$$

This inequality shows that if the maximum error $\kappa_{1}-\bar{\lambda}_{1}$ is small compared with the interval $\bar{\lambda}_{2}-\bar{\lambda}_{1}$, the difference $w_{1}-u_{1}$ is small in norm. The bound (1.6) is sharp in the sense that equality is attained when $\lambda_{i}=\bar{\lambda}_{i}$ and $a_{i}=0$ for $i>2$. The inequality (1.6) is trivial for $\kappa_{1} \geq \bar{\lambda}_{2}$.

In this paper we generalize the bound (1.6). We give a bound for the norm of $w_{p}-u_{p}$ in terms of the given bounds $\kappa_{i}$ and $\bar{\lambda}_{i}$. This bound is again sharp in the sense that equality may be attained. The bound for $\left(w_{p}-u_{p}, w_{p}-u_{p}\right)$ is small if the maximum error $\kappa_{p}-\bar{\lambda}_{p}$ is small relative to both $\bar{\lambda}_{p+1}-\kappa_{p}$ and $\bar{\lambda}_{p}-\kappa_{p-1}$. It becomes trivial if $\kappa_{p} \geq \bar{\lambda}_{p+1}$ or $\kappa_{p-1} \geq \bar{\lambda}_{p}$. The case $p=1$ gives an improved but more complicated version of (1.6).

If $\lambda_{p}$ is multiple eigenvalue, we can only expect $w_{p}$ to approximate one of the associated eigenvectors. Hence if $\lambda_{p}$ lies near to several other eigenvalues we must expect $w_{p}$ to approximate not $u_{p}$ but a linear combination of the eigenvectors corresponding to the nearby eigenvalues. This approximation is established in section 3 .

It is possible to find a bound for $\left(w_{p}-u_{p}, w_{p}-u_{p}\right)$ by determining to what extent $w_{p}$ satisfies the eigenvalue equation. Such bounds, which involve $\left(A w_{p}-\kappa_{p} w_{p}, A w_{p}-\kappa_{p} w_{p}\right)$ have been found by several authors [12, 20,23]. Our bound, however, involves only the $\kappa_{i}$ and the lower bounds $\bar{\lambda}_{i}$. It should be particularly useful in the case of differential operators where the Rayleigh-Ritz trial functions may not be sufficiently differentiable to give a finite value of $\left(A w_{p}, A w_{p}\right)$. Our bounds are established by algebraic means for the case when $A$ is an $N \times N$ matrix. They are independent of $N$. Consequent- 
ly, the bounds also hold for infinite-dimensional operators $A$ whose first $p$ eigenvalues and eigenvectors are approximated uniformly by those of a sequence $A_{N}$ of $N \times N$ matrices. This is certainly the case if $A$ is completely continuous. It also holds under the weaker condition that $A$ have $p$ discrete eigenvalues defined by a minimum maximum principle. These must lie below any continuous spectrum. Thus $A$ may be a Schroedinger operator corresponding to both bound and unbound states.

The fact that the eigenvalues $\lambda_{i}$ are stationary values of the Rayleigh quotient tends to make the approximation of the eigenvectors worse than that of the eigenvalues. In fact, the bound (2.41) shows that the square of the norm of the error $w_{p}-u_{p}$ is of the order $\kappa_{p}-\overline{\lambda_{p}}$.

The error bounds in sections 2 and 3 are in the sense of the norm. If $A$ is a differential operator, its eigenvectors $u_{i}$ are functions. It is often of interest to approximate the value of the function at a particular point. An adaptation of the method of Diaz and Greenberg [7,9] which leads to such a pointwise approximation is presented in section 4 .

\section{Separated Eigenvalues}

Let $A$ be an hermitian $N \times N$ matrix. It is a linear operator on Euclidean $N$-space. Let the usual scalar product between two vectors $u$ and $v$ on this space be denoted by $(u, v)$.

Let the eigenvalues of $A$ be $\lambda_{1} \leq \lambda_{2} \leq \ldots \leq \lambda_{N}$. The corresponding eigenvectors are denoted by $u_{1}, \ldots, u_{N}$ so that the equations

$$
A u_{i}=\lambda_{i} u_{i} \quad i=1, \ldots, N
$$

are satisfied. The $u_{i}$ are normalized so that

$$
\left(u_{i}, u_{j}\right)=\delta_{i j} \quad i, j=1, \ldots, N .
$$

Then (2.1) implies

$$
\left(A u_{i}, u_{j}\right)=\lambda_{i} \delta_{i j} \quad i, j=1, \ldots, N .
$$

We suppose that the Rayleigh-Ritz method $[1,5,19]$ is applied to find upper bounds

$$
\kappa_{\alpha} \geq \lambda_{\alpha} \quad \alpha=1, \ldots, M
$$

for the first $M<N$ eigenvalues of $A$. This is done by choosing $M$ linearly independent vectors $v_{1}, \ldots, v_{M}$ and finding the roots $\kappa_{1} \leq \kappa_{2} \leq \ldots . \kappa_{M}$ of the secular equation

$$
\operatorname{det}\left[\left(A v_{\alpha}, v_{\beta}\right)-\kappa\left(v_{\alpha}, v_{\beta}\right)\right]=0 \quad \alpha, \beta=1, \ldots, M .
$$

Associated with each of the $\kappa_{\alpha}$ is a linear combination

$$
w_{\alpha}=\sum_{\beta=1}^{M} C_{\alpha}^{\beta} v_{\beta}
$$

of unit length such that the $M$ linear equations

$$
\left(A w_{\alpha}, v_{\beta}\right)=\kappa_{\alpha}\left(w_{\alpha}, v_{\beta}\right) \quad \beta=1, \ldots, M .
$$

are satisfied. Then

and

$$
\left(w_{\alpha}, w_{\beta}\right)=\delta_{\alpha \beta}
$$

$$
\left(A w_{\alpha}, w_{\beta}\right)=\kappa_{\alpha} \delta_{\alpha \beta} \quad \alpha, \beta=1, \ldots, M .
$$

We assume that in addition to the upper bounds $\kappa_{\alpha}$ we have determined some lower bounds $\bar{\lambda}_{i}$ such that

$$
\lambda_{i} \geq \bar{\lambda}_{i} \quad i=1, \ldots, N .
$$

This means that we have at least some idea of the degree of approximation of the $\kappa_{\alpha}$ to the eigenvalues $\lambda_{\alpha}$

We seek to determine from this degree of approximation of the $\kappa_{\alpha}$ to the eigenvalues the degree of approximation of a particular Rayleigh-Ritz eigenvector $w_{p}$ to the eigenvector $u_{p}$ of $A$.

We assume that $\lambda_{\underline{p-1}}<\lambda_{p}<\lambda_{p+1}$ and that the bounds $\kappa_{p-1}, \kappa_{p}, \bar{\lambda}_{p}$, and $\bar{\lambda}_{p+1}$ are sufficiently good that

and

$$
\kappa_{p}<\bar{\lambda}_{p+1}
$$

$$
\kappa_{p-1}<\bar{\lambda}_{p} \text {. }
$$

Our problem is the following: Given the $M$ vectors $w_{1}, \ldots, w_{M}$ satisfying (2.8), find the largest value of the deviation $\left(w_{p}-u_{p}, w_{p}-u_{p}\right)$ of $w_{p}$ from the $p$ th normalized eigenvector of any matrix $A$ satisfying the eq (2.9) and having eigenvalues $\lambda_{i}$ satisfying $(2.10)$.

If the eigenvalues $\lambda_{i}$ of $A$ are given, $A$ is completely specified by prescribing its normalized eigenvectors. These eigenvectors $u_{1}, \ldots, u_{N}$ form a basis, so that the $w_{\alpha}$ can be written as linear combinations of them. Let

$$
w_{\alpha}=\sum_{i=1}^{N} a_{\alpha}^{i} u_{i} \quad \alpha=1, \ldots, M
$$

Since the $u_{i}$ satisfy (2.2) and (2.3), the eqs (2.8) and (2.9) become

$$
\sum_{i=1}^{N} a_{\alpha}^{i} a_{\beta}^{i}=\delta_{\alpha \beta}
$$

and

$$
\sum_{i=1}^{N} \lambda_{i} a_{\alpha}^{i} a_{\beta}^{i}=\kappa_{\alpha} \delta_{\alpha \beta} \quad \alpha, \beta=1, \ldots, M
$$

On the other hand,

$$
\left(w_{p}-u_{p}, w_{p}-u_{p}\right)=2\left(1-a_{p}^{p}\right) .
$$

If $u_{p}$ satisfies (2.2) and (2.3), so does $-u_{p}$. We choose the sign to make $a_{p}^{p}$ non-negative. Thus, our problem of maximizing $\left(w_{p}-u_{p}, w_{p}-u_{p}\right)$ is reduced to that of minimizing $\left(a_{p}^{p}\right)^{2}$ under the constraints (2.14), (2.15), and (2.10). 
We first keep the eigenvalues $\lambda_{i}$ fixed and unequal. If $a_{\alpha}^{i}$ is the minimizing set of coefficients, we find by direct differentiation that the equations

$$
\begin{array}{rl}
a_{p}^{p} \delta_{i p} \delta_{\alpha p}+\sum_{\beta=1}^{M}\left(r_{\alpha \beta}+\lambda_{i} s_{\alpha \beta}\right) a_{\beta}^{i}=0 & i=1, \ldots, N \\
\alpha & =1, \ldots, M
\end{array}
$$

must be satisfied. The $r_{\alpha \beta}$ and $s_{\alpha \beta}$ are Lagrange multipliers, and are symmetric:

$$
\begin{aligned}
& r_{\alpha \beta}=r_{\beta \alpha}, \\
& s_{\alpha \beta}=s_{\beta \alpha} .
\end{aligned}
$$

To solve the eq (2.17), we multiply by $a_{\gamma}^{i}$ and sum with respect to $i$. Using (2.14) and (2.15) we find that

$$
a_{p}^{p} a_{\gamma}^{p} \delta_{\alpha p}+r_{\alpha \gamma}+\kappa_{\gamma} s_{\alpha \gamma}=0 .
$$

Interchanging $\alpha$ and $\gamma$ and using (2.18), we find $r_{\alpha \gamma}=-\left(\kappa_{\alpha}-\kappa_{\gamma}\right)^{-1} \kappa_{p} a_{p}^{p}\left[a_{\gamma}^{p} \delta_{\alpha p}-a_{\alpha}^{p} \delta_{\gamma p}\right]$, $s_{\alpha \gamma}=\left(\kappa_{\alpha}-\kappa_{\gamma}\right)^{-1} a_{p}^{p}\left[a_{\gamma}^{p} \delta_{\alpha p}-a_{\alpha}^{p} \delta_{\gamma p}\right] \quad \alpha \neq \gamma$.

In particular, $r_{\alpha \gamma}$ and $s_{\alpha \gamma}$ vanish unless $\alpha=\gamma$ or either $\alpha$ or $\gamma$ is $p$.

Letting $\alpha=\gamma$ in (2.19), we find

$$
r_{\alpha \alpha}+\kappa_{\alpha} s_{\alpha \alpha}=-\left(a_{p}^{p}\right)^{2} \delta_{\alpha p} .
$$

We substitute (2.20) and (2.21) in (2.17) to obtain $\left(\kappa_{p}-\kappa_{\alpha}\right)\left(\lambda_{i}-\kappa_{\alpha}\right) s_{\alpha \alpha} a_{\alpha}^{i}+\left(\lambda_{i}-\kappa_{p}\right) a_{p}^{p} a_{\alpha}^{p} a_{p}^{i}=0 \quad \alpha \neq p$

and

$$
\begin{array}{r}
a_{p}^{p} \delta_{i p}+\left[\left(\lambda_{i}-\kappa_{p}\right) s_{p p}-\left(a_{p}^{p}\right)^{2}\right] \\
a_{p}^{i}-\sum_{\beta \neq p} \frac{\lambda_{i}-\kappa_{p}}{\kappa_{\beta}-\kappa_{p}} a_{p}^{p} a_{\beta}^{p} a_{\beta}^{i} \\
=0, \quad i=1, \ldots, N .
\end{array}
$$

If the value $a_{n}^{p}=0$ is compatible with the constraints (2.14) and (2.15), the minimum value of $\left(a_{p}^{p}\right)^{2}$ is clearly zero. We suppose for the moment that this is not the case, so that

$$
a_{p}^{p} \neq 0 .
$$

Then we can solve $(2.23)$ with $i=p$ for $s_{p p}$ in terms of the $a_{\beta}^{p}$. We can also eliminate $s_{\alpha \alpha}$ between (2.22) with $i=p$ and any other value of $i$ to obtain

$$
a_{p}^{p} a_{\beta}^{p} a_{\beta}^{i}=\frac{\left(\lambda_{i}-\kappa_{p}\right)\left(\lambda_{p}-\kappa_{\beta}\right)}{\left(\lambda_{p}-\kappa_{p}\right)\left(\lambda_{i}-\kappa_{\beta}\right)} a_{p}^{i}\left(a_{\beta}^{p}\right)^{2} .
$$

Substituting this and the value of $s_{p p}$ in (2.23) we obtain

$$
\begin{array}{r}
a_{p}^{i}\left\{\frac{\lambda_{i}-\kappa_{p}}{\lambda_{p}-\kappa_{p}}\left[1-\sum_{\beta=1}^{M}\left(a_{\beta}^{p}\right)^{2}+\sum_{\beta=1}^{M} \frac{\lambda_{p}-\kappa_{\beta}}{\lambda_{i}-\kappa_{\beta}}\left(a_{\beta}^{p}\right)^{2}\right]\right\} \\
=0, \quad i \neq p .
\end{array}
$$

Suppose now that exactly $L$ of the coefficients $a_{\beta}^{p}$ are nonzero:

$$
\begin{aligned}
& a_{\beta}^{p} \neq 0 \quad \text { for } \quad \beta=\beta_{1}, \beta_{2}, \ldots, \beta_{L} \\
& \beta_{1}<\beta_{2}<\ldots<\beta_{L} .
\end{aligned}
$$

By (2.24) one of the $\beta_{\nu}=p$. The term in braces in (2.26) times the product of the $\left(\lambda_{i}-\kappa_{\beta_{\nu}}\right)$ with $\beta_{\nu} \neq p$ is a polynomial of degree $L$ in $\lambda_{i}$. Hence it vanishes for at most $L$ values of $i$. Consequently, $a_{p}^{i} \neq 0$ for $\widehat{L} \leq L$ values of $i \neq p$.

From (2.22) it follows that $a_{p}^{i}=0$ implies $s_{\alpha \alpha} a_{\alpha}^{i}=0$ for all $\alpha$. But $s_{\alpha \alpha}=0$ implies $a_{\alpha}^{p}=0$. Hence $a_{p}^{i}=0$ implies $a_{\beta_{\nu}}^{i}=0$. Thus our vectors $w_{\alpha}$ split into two subsets: The subset $S_{1}$ consists of $L$ orthonormal vectors $w_{\beta_{1}}$, . . ., $w_{\beta_{L}}$ (including $w_{p}$ ) having only components in the $\bar{L}+1$ directions $u_{i_{1}}$. . ., $u_{i_{L}+1}$. The subset $S_{2}$ consists of $M-\bar{L}-1$ vectors orthogonal to $u_{p}$.

It follows that $\bar{L}$ must be either $L$ or $L-1$. In the latter case, the $L$ vectors $w_{\beta_{\nu}}$ in the first set will be the eigenvectors $u_{i_{1}}, \ldots, u_{i_{L}}$ themselves. In particular, $w_{p}$ is $u_{p}$ so that the corresponding maximum of $\left(w_{p}-u_{p}, w_{p}-u_{p}\right)$ is zero. This occurs if and only if $\kappa_{p}=\lambda_{p}$. The more interesting case is that in which

$$
\bar{L}=L \text {. }
$$

The term in the braces in (2.26) vanishes for $i=i_{1}, i_{2}, \ldots, i_{L+1}\left(i_{1}<i_{2}<\ldots .<i_{L+1}\right)$ except when $i_{\nu}=p$. This represents a set of $L$ linear equations in the $L$ unknowns $\left(a_{\beta_{1}}^{p}\right)^{2} \ldots,\left(a_{\beta_{L}^{P}}\right)^{2}$. It can be solved explicitly to yield

$$
\left(a_{\beta_{\mu}}^{p}\right)^{2}=\prod_{\substack{\nu=1 \\ \nu \neq \mu}}^{L} \frac{\lambda_{p}-\kappa_{\beta_{\nu}}}{\kappa_{\beta_{\mu}}-\kappa_{\beta_{\nu}}} \underset{\substack{\tau=1 \\ i_{\tau} \neq p}}{L+1} \frac{\kappa_{\beta_{\mu}}-\lambda_{i_{\tau}}}{\lambda_{p}-\lambda_{i_{\tau}}}
$$

(This result can be checked by the Lagrange interpolation formula [17]. A similar solution of a closely related equation was given by $\mathrm{K}$. Loewner, Math. Z. 38, 180-181 (1934).)

We now let $\beta=\beta_{\mu}$ in (2.25), multiply by $a_{p}^{i}$, and sum. By (2.14) we have

$$
\sum_{i_{\tau} \neq \rho} \frac{\lambda_{i_{\tau}}-\kappa_{\rho}}{\lambda_{i_{\tau}}-\kappa_{\beta_{\mu}}}\left(a_{p}^{i_{\tau}}\right)^{2}+\frac{\lambda_{p}-\kappa_{p}}{\lambda_{p}-\kappa_{\beta \mu}}\left(a_{\rho}^{p}\right)^{2}=\delta_{p \beta_{\mu}} \quad \mu=1, \ldots, L .
$$

Again, we can solve explicitly for the $L$ unknowns $\left(a_{p}^{i \tau}\right)^{2}, i_{\tau} \neq p$. Using (2.25) and (2.29), we find that

$$
\left(a_{\beta_{\mu}}^{i_{\tau}}\right)^{2}=\prod_{\substack{\nu=1 \\ \nu \neq \mu}}^{L} \frac{\lambda_{i_{\sigma}}-\kappa_{\beta_{\nu}}}{\kappa_{\beta_{\mu}}-\kappa_{\beta_{\nu}}} \underset{\substack{\tau=1 \\ \tau \neq \sigma}}{I+1} \frac{\kappa_{\beta_{\mu}}-\lambda_{i_{\tau}}}{\lambda_{i_{\sigma}}-\lambda_{i_{\tau}}}
$$


The solution (2.31) formally satisfies the conditions (2.14), (2.15), and (2.17). (The square roots must be chosen so that a $\beta_{\beta_{\mu}}^{i \sigma}$ is $\left(\lambda_{i_{\sigma}}-\kappa_{\beta_{\mu}}\right)^{-1}$ times a function of $i_{\sigma}$ only times a function of $\beta_{v}$ only.) In order to be admissible, the coefficients must be real. It is easily seen that this is the case if and only if

$$
\lambda_{i_{1}}<\kappa_{\beta_{1}}<\lambda_{i_{2}}<\ldots<\kappa_{\beta_{L}}<\lambda_{i_{L+1}} .
$$

The vectors $w_{\beta}$ in the subset $S_{2}$ are orthogonal to $w_{\beta_{1}}, \ldots, w_{\beta_{L}}$ and $u_{p}$. It follows from (2.31) that they are orthogonal to $u_{i_{1}}, \ldots, u_{i_{L+1}}$. This is possible if and only if to each $i$ with $i \neq i_{1}, \ldots ., i_{L+1}$ there corresponds a separate $\kappa_{\beta}$ with $\beta \neq \beta_{1}, \ldots$. ., $\beta_{L}$ such that $\kappa_{\beta} \geq \lambda_{i}$.

We now consider the possible minima of $\left(a_{p}^{p}\right)^{2}$. Choosing a particular set of $\beta_{\nu}$ and $i_{\sigma}$, we find from (2.31) that

$$
\left(a_{p}^{p}\right)^{2}=\prod_{\boldsymbol{\beta}_{\nu \neq p} \neq} \frac{\lambda_{p}-\kappa_{\boldsymbol{\beta}_{\nu}}}{\kappa_{p}-\kappa_{\boldsymbol{\beta}_{\nu}}} \prod_{i_{\tau} \neq p} \frac{\kappa_{p}-\lambda_{i_{\tau}}}{\lambda_{p}-\lambda_{i_{\tau}}}
$$

Because of (2.32)

$$
\frac{\left(\lambda_{p}-\kappa_{\boldsymbol{\beta}_{\nu}}\right)\left(\kappa_{p}-\lambda_{i_{v}}\right)}{\left(\kappa_{p}-\kappa_{\boldsymbol{\beta}_{\nu}}\right)\left(\lambda_{p}-\lambda_{i_{v}}\right)}=1-\frac{\left(\kappa_{p}-\lambda_{p}\right)\left(\kappa_{\boldsymbol{\beta}_{\nu}}-\lambda_{i_{v}}\right)}{\left(\kappa_{p}-\kappa_{\boldsymbol{\beta}_{\nu}}\right)\left(\lambda_{p}-\lambda_{i_{\nu}}\right)}<1
$$

for $i_{\nu}>p$ or $\beta_{\nu}<p$.

Thus, the right-hand side of (2.33) is increased by dropping any pair $\lambda_{i_{y}}, \kappa_{\beta_{\nu}}$. This means that its minimum will be attained when the sets $i_{1}$. . ., $i_{L+1}, \beta_{1}$. . ., $\beta_{L}$ are maximal with respect to the properties required of them. We further note that the right-hand side of (2.33) is an increasing function of $\lambda_{i_{\tau}}$ for $i_{\tau} \neq p$ and a decreasing function of $\kappa_{\beta_{y}}$ for $\boldsymbol{\beta}_{\nu} \neq p$.

Keeping these facts in mind, we construct the sets $i_{1} . . ., i_{L+1}, \beta_{1}, \ldots ., \beta_{L}$ which minimize $\left(a_{p}^{p}\right)^{2}$ for fixed unequal $\lambda_{i}$ as follows.

Let

$$
i_{1}=1 \text {. }
$$

Let

$$
i_{2}=\min \left\{i \mid \boldsymbol{H} \beta \ni \lambda_{1}<\kappa_{\beta}<\lambda_{i}\right\} \text {. }
$$

Let

$$
\beta_{1}=\max \left\{\left.\beta\right|_{\kappa_{\beta}}<\lambda_{i_{2}}\right\} .
$$

Then inductively, let

$$
\begin{aligned}
i_{\nu+1} & =\min \left\{i \mid \boldsymbol{H}_{\kappa_{\beta}} \ni \lambda_{i_{\nu}}<\kappa_{\beta}<\lambda_{i}\right\}, \\
\beta_{\nu} & =\max \left\{\beta \mid \kappa_{\beta}<\lambda_{i_{\nu+1}}\right\} .
\end{aligned}
$$

Because of (2.11) the set of $\beta_{\nu}$ includes $p$. If $p$ is not included in the $i_{\sigma}$, we can easily construct a solution of the eqs (2.14) and (2.15) with $a_{p}^{p}=0$. To do this we define the $\left(a_{\beta_{\mu}^{\alpha}}^{i}\right)^{2}$ by $(2.31)$. Then $a_{p}^{p}=\left(w_{p}, u_{p}\right)=0$.

Conversely, if (2.24) is violated so that the minimum of $\left(a_{p}^{p}\right)^{2}$ is zero, we can consider the problem of minimizing a coefficient $\left(a_{p}^{q}\right)^{2}$ with $q<p$ under the constraints (2.14), (2.15), and $a_{p}^{i}=0$ for some set of $i<p$ including $p$. Since $\kappa_{p}<\lambda_{p+1}$, not all the $a_{p}^{q}$ can vanish. Therefore this minimum problem will have a non-zero solution for some $q$ and some set of $i \leq p$. The minimizing conditions again lead to the determination of sets $i_{\sigma}$ and $\beta_{\nu}$ by (2.35), (2.36), (2.37), and (2.38). The integer $p$ is included in the $\beta_{\nu}$ but not in the $i_{\sigma}$.

Thus, $a_{p}^{p} \neq 0$ if and only if $p$ is one of the $i_{\sigma}$. It follows from the construction of the $i_{\sigma}$ that this will be the case if and only if there is a $\kappa_{\beta}$ such that $\lambda_{p-1}<\kappa_{\beta}<\lambda_{p}$. This is assured by (2.12) for any $\lambda_{i}$ satisfying (2.10). Condition (2.12) is therefore necessary and sufficient for $\left(a_{p}^{p}\right)^{2}$ to have a nonzero minimum.

The minimum value of $\left(a_{p}^{p}\right)_{2}$ is now given by (2.33). It is a continuous nondecreasing function of the eigenvalues $\lambda_{i}$. Hence its minimum with respect to the $\lambda_{i}$ satisfying (2.10) will occur for $\lambda_{i}=\bar{\lambda}_{i}$. We may remove the assumption that the $\lambda_{i}$ are unequal by a limiting process. This will alter the inequalities in (2.37) and (2.38) slightly.

As we pointed out in the introduction, we can replace the matrix $A$ by a symmetric operator on a Hilbert space. We need only assume that $A$ has at least $p$ discrete eigenvalues defined by a minimum maximum principle $[1,5,19]$. For then the first $N$ eigenvalues of $A$ and their corresponding eigenvectors are uniformly approximated by those of an $N^{\prime} \times N^{\prime}$ matrix $A_{N^{\prime}}$ for $N^{\prime}$ sufficiently large. If $\bar{\lambda}_{i} i=1, \ldots ., N$ are lower bounds for the first $N$ eigenvalues of $A$, there is an $\epsilon_{N^{\prime}}$ which goes to zero as $N^{\prime} \rightarrow \infty$ such that $\bar{\lambda}_{i}-\epsilon_{N^{\prime}}$ are lower bounds for the first $N$ eigenvalues of $A_{N^{\prime}}$. Also, $\overline{\lambda_{N}}-\epsilon_{N^{\prime}}$ is a lower bound for the higher eigenvalues of $A_{N^{\prime}}$. We let $N^{\prime} \rightarrow \infty$ for fixed $M$ and $N$. Using (2.33) and (2.34), we obtain the following theorem.

THEOREM 1. Let $\kappa_{1} \leq \kappa_{2} \leq . . \leq \kappa_{M}$ be the Rayleigh-Ritz upper bounds for the first $\mathrm{M}$ of the eigenvalues $\lambda_{1} \leq \lambda_{2} \leq$. . . of a symmetric linear operator $A$. Let $\bar{\lambda}_{1} \leq \ldots . \leq \bar{\lambda}_{N}$ be lower bounds for the first $N$ eigenvalues of $A, \bar{N} \geq M$.

Let

$$
\kappa_{p-1}<\bar{\lambda}_{p}<\kappa_{p}<\bar{\lambda}_{p+1} .
$$

Define the numbers

$$
\begin{aligned}
i_{1}<i_{2}<\ldots<i_{L+1}, \quad \beta_{1}<\beta_{2}<\ldots<\beta_{L} \text { by } \\
i_{1}=1 \\
i_{2}=\min \left\{i \mid \bar{\lambda}_{i}>_{\kappa_{1}}\right\}
\end{aligned}
$$




$$
\begin{aligned}
\beta_{1} & =\max \left\{\beta \mid \kappa_{\beta} \leq \bar{\lambda}_{i_{2}}\right\} \\
i_{\nu+1} & =\min \left\{i \mid \mathbb{H} \beta_{\ni} \bar{\lambda}_{i_{\nu}}<\kappa_{\beta}<\bar{\lambda}_{i}\right\} \\
\beta_{\nu} & =\max \left\{\beta \mid \kappa_{\beta} \leq \bar{\lambda}_{i_{\nu+1}}\right\}
\end{aligned}
$$

Then if $w_{p}$ is the normalized Rayleigh-Ritz eigenvector corresponding to the bound $\kappa_{p}$ and $u_{p}$ is the normalized eigenvector of $A$ corresponding to the eigenvalue $\lambda_{p}$,

$$
\begin{aligned}
& {\left[1-\frac{1}{2}\left(w_{p}-u_{p}, w_{p}-u_{p}\right)\right]^{2}}
\end{aligned}
$$

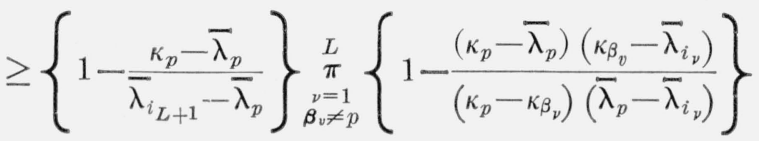

The right-hand side of this inequality approaches zero if either $\kappa_{p}$ approaches $\bar{\lambda}_{p+1}$ or $\kappa_{p-1}$ approaches $\bar{\lambda}_{p}$. It is near one if the error $\kappa_{p}-\bar{\lambda}_{p}$ is small relative to the approximate spacing $\bar{\lambda}_{i_{L+1}}-\bar{\lambda}_{p}$ and if the products of errors $\left(\kappa_{p}-\bar{\lambda}_{p}\right)\left(\kappa_{\beta_{\nu}}-\bar{\lambda}_{i_{\nu}}\right)$ are small relative to the products of approximate spacings $\left(\kappa_{p}-\kappa_{\beta_{\nu}}\right)\left(\bar{\lambda}_{p}-\bar{\lambda}_{i_{\nu}}\right)$.

If lower bounds $\bar{\lambda}_{i}$ are not given for all the eigenvalues $\lambda_{i}$, we can always use a lower bound for any particular eigenvalue as a lower bound for a higher eigenvalue. In particular, we can let $\bar{\lambda}_{i}=\bar{\lambda}_{p+1}$ for $i>p$ and $\overline{\lambda_{i}}=\overline{\lambda_{1}}$ for $i<p$. If $p>1,(2.39)$ requires that $\kappa_{p-1}<\bar{\lambda}_{p}$. Then $L=2, i_{1}=1, \quad \beta_{1}=p-1, i_{2}=\beta_{2}=p$, $i_{3}=p+1$. This leads to the simpler bound

$$
\begin{aligned}
& {\left[1-\frac{1}{2}\left(w_{p}-u_{p}, w_{p}-u_{p}\right)\right]^{2}}
\end{aligned}
$$

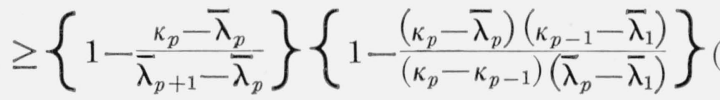

Even though this bound has fewer factors than (2.41), it is, in general, smaller.

For $p=1$ we can take $\bar{\lambda}_{i}=\bar{\lambda}_{2}$ for $i \geq 2$. Then $L=1$, $i_{1}=\beta_{1}=1, i_{2}=2$, and (2.41) reduces to (1.6).

Example. We apply the Rayleigh-Ritz method to the matrix

$$
A=\left(\begin{array}{rrr}
1 & 1 & 0 \\
1 & 3 & 1 \\
0 & 1 & 23
\end{array}\right)
$$

using trial vectors with vanishing third component. This amounts to finding the eigenvalues and eigenvectors of the $2 \times 2$ matrix obtained by striking out the third row and column of $A$. We obtain

$$
\kappa_{1}=2-\sqrt{2}, \kappa_{2}=2+\sqrt{2}
$$

with the corresponding vectors

$$
\begin{aligned}
& w_{1} \sim\left(\frac{1}{2}(2+\sqrt{2})^{1 / 2}, \frac{1}{2}(2-\sqrt{2})^{1 / 2}, 0\right) \\
& w_{2} \sim\left(\frac{1}{2}(2-\sqrt{2})^{1 / 2}, \frac{1}{2}(2+\sqrt{2})^{1 / 2}, 0\right)
\end{aligned}
$$

If the first two coordinate directions are replaced by the $w_{1}$ and $w_{2}$ directions, the matrix $A$ "becomes

$$
A^{*}=\left(\begin{array}{ccc}
\kappa_{1} & 0 & -\frac{1}{2} \kappa_{1}{ }^{1 / 2} \\
0 & \kappa_{2} & -\frac{1}{2} \kappa_{2}{ }^{1 / 2} \\
-\frac{1}{2} \kappa_{1}{ }^{1 / 2} & -\frac{1}{2} \kappa_{2}{ }^{1 / 2} & 23
\end{array}\right)
$$

We now obtain lower bounds for the eigenvalues by means of Hadamard's theorem ion Ideterminants [16].

$$
\begin{aligned}
& \bar{\lambda}_{1}=\kappa_{1}-\frac{1}{2} \kappa_{1}^{1 / 2}, \\
& \bar{\lambda}_{2}=\kappa_{2}-\frac{1}{2} \kappa_{2}{ }^{1 / 2} .
\end{aligned}
$$

For $\bar{\lambda}_{3}$ we use the fact that the largest eigenvalue must exceed the largest diagonal element. Then

$$
\bar{\lambda}_{3}=23 \text {. }
$$

Substituting these values in (2.41) we find that

$$
\begin{gathered}
\left(w_{1}-u_{1}, w_{1}-u_{1}\right) \leq 0.08025, \\
\left(w_{2}-u_{2}, w_{2}-u_{2}\right) \leq 0.09980 .
\end{gathered}
$$

In this problem one can, of course, determine the eigenvalues and the corresponding eigenvectors explicitly. We find that

$$
\begin{aligned}
& \left(w_{1}-u_{1}, w_{1}-u_{1}\right)=0.000328, \\
& \left(w_{2}-u_{2}, w_{2}-u_{2}\right)=0.002238 .
\end{aligned}
$$

Thus, the error bounds (2.49) are a good deal larger than the errors themselves.

Our error bounds depend upon the lower bounds $\bar{\lambda}_{i}$. These were chosen rather crudely and could be improved in various ways (see, for example [5]). In order to determine the effect of such an improvement, we replace the $\bar{\lambda}_{i}$ by the eigenvalues $\lambda_{i}$ in (2.41). We then obtain the bounds

$$
\begin{aligned}
& \left(w_{1}-u_{1}, w_{1}-u_{1}\right) \leq 0.000330, \\
& \left(w_{2}-u_{2}, w_{2}-u_{2}\right) \leq 0.002243 .
\end{aligned}
$$


These are very close to the actual values (2.50).

The simpler bound (1.6) gives

$$
\left(w_{1}-u_{1}, w_{1}-u_{1}\right) \leq 0.195136
$$

if the values (2.47) of $\bar{\lambda}_{i}$ are used, and

$$
\left(w_{1}-u_{1}, w_{1}-u_{1}\right) \leq 0.002364
$$

if they are replaced by the $\lambda_{i}$ themselves. In both cases we see that the bound (2.41) is significantly better than the simplified bound (1.6).

\section{Neighboring Eigenvalues}

The condition (2.39) implies that the eigenvalue $\lambda_{p}$ is simple. If this is not the case, the corresponding eigenvector $u_{p}$ is not uniquely defined. In fact, if $\lambda_{p}$ has multiplicity $m, u_{p}$ may be any element of an $m$-space. If $m>1$, there will always be such a $u_{p}$ orthogonal to $w_{p}$, so that the minimum of $\left(w_{p}, u_{p}\right)$ is zero.

We must reformulate our problem. We seek the minimum value of $\left(w_{p}, u_{p}\right)^{2}$ when $u_{p}$ is taken to be that element of the $m$-space which best approximates $w_{p}$. This $u_{p}$ is the projection of $w_{p}$ into the $m$-space of eigenvectors corresponding to $\lambda_{p}$.

The condition (2.39) implies not only that $\lambda_{p}$ is simple, but that our bounds $\kappa_{p-1}, \kappa_{p}, \bar{\lambda}_{p}$, and $\bar{\lambda}_{p+1}$ are good enough to reveal its simplicity. That is, the error in our bounds is smaller than the separations between $\lambda_{p-1}, \lambda_{p}$, and $\lambda_{p+1}$. If this is not the case, we cannot distinguish between a simple and a multiple eigenvalue.

Suppose now that the upper and lower bounds for $\lambda_{p}, \lambda_{p+1}, \ldots, \lambda_{p+m-1}$ show these eigenvalues to lie close together. Suppose further that

$$
\kappa_{p-1},<<\bar{\lambda}_{p}, \kappa_{p+m-1}<<\bar{\lambda}_{p+m}
$$

so that $\lambda_{p-1}$ and $\lambda_{p+m}$ are known to lie away from the cluster of eigenvalues about $\lambda_{p}$. Then, to our degree of approximation $\lambda_{p}$ behaves like an eigenvalue of multiplicity $m$. We ask how well $w_{p}$ can be approximated by a linear combination of unit length of the eigenvectors $u_{p}, u_{p+1}, \ldots, u_{p+m-1}$.

This problem is equivalent to that of minimizing $\sum_{p}^{p+m-1}\left(a_{p}^{i}\right)^{2}$ under the constraints $(2.14),(2.15)$, and (2.10). By (2.20) we have $r_{\alpha \beta}=s_{\alpha \beta}=0$ unless $\alpha=\beta$ or $\alpha$ or $\beta=p$. Moreover, $r_{\alpha p}+\kappa_{p} s_{\alpha p}=0, r_{\alpha \alpha}+\kappa_{\alpha} s_{\alpha \alpha}=0$ for $\alpha \neq p$. This means that we would obtain the same minimizing conditions by imposing only the constraints (2.14) with $\alpha=\beta$ or $\alpha$ or $\beta=p$ and the single constraint (2.15) with $\alpha=\beta=p$. The latter may even be replaced by the inequality

$$
\sum_{i=1}^{N} \lambda_{i}\left(a_{p}^{i}\right)^{2} \leq \kappa_{p}
$$

The other constraints (2.14) and (2.15) determine which local minima actually occur, but the local minima themselves are determined by (2.14) and (3.2).

The same situation applies in the case under consideration here. Necessary conditions for a maximum of $\sum_{p}^{p+m-1}\left(a_{p}^{i}\right)^{2}$ are determined by the constraints (2.14), (2.10), and (3.2).

Let $\tilde{u}_{p}$ be the unit vector in the direction of the projection of $w_{p}$ into the space spanned by $u_{p}, \ldots$, $u_{p+m-1}$ :

$$
\tilde{u}_{p}=\left\{\sum_{i=p}^{p+m-1}\left(a_{p}^{i}\right)^{2}\right\}^{-1 / 2} \sum_{p}^{p+m-1} a_{p}^{i} u_{i}
$$

Let $\tilde{u}_{p+1}, \ldots ., \tilde{u}_{p+m-1}$ be other linear combinations of $u_{p}, \ldots, u_{p+m-1}$ such that $\tilde{u}_{p}, \ldots, \tilde{u}_{p+m-1}$ are orthonormal. Let $\tilde{u}_{i}=u_{i}$ for $i \neq p, \ldots, p+m-1$ and put

$$
\tilde{a}_{\alpha}^{i}=\left(w_{\alpha}, \tilde{u}_{i}\right) .
$$

Then by construction

and

$$
\tilde{a}_{p}^{i}=0 \quad i=p+1, \ldots, p+m-1
$$

$$
\left(\tilde{a}_{p}^{p}\right)^{2}=\sum_{i=p}^{p+m-1}\left(a_{p}^{i}\right)^{2}
$$

Moreover,

$$
\sum_{i=1}^{N} \lambda_{i}\left(a_{p}^{i}\right)^{2} \geq \sum_{1}^{p-1} \lambda_{i}\left(\tilde{a}_{p}^{i}\right)^{2}+\lambda_{p}\left(\tilde{a}_{p}^{p}\right)^{2}+\sum_{p+m}^{N} \lambda_{i}\left(\tilde{a}_{p}^{i}\right)^{2} .
$$

Thus, if we let

$$
\tilde{\lambda}_{i}= \begin{cases}\lambda_{p} & i=p, \ldots, p+m-1 \\ \lambda_{i} & \text { otherwise. }\end{cases}
$$

We have from (3.2) and (3.7) that

$$
\sum_{1}^{N} \tilde{\lambda}_{i}\left(\tilde{a}_{p}^{i}\right)^{2} \leq \kappa_{p}
$$

Since the $\tilde{u}_{i}$ are orthonormal, (2.14) becomes

$$
\sum \tilde{a}_{\alpha}^{i} \tilde{a}_{\beta}^{i}=\delta_{\alpha \beta}
$$

Our problem is thus reduced to minimizing $\left(\tilde{a}_{p}^{p}\right)^{2}$ under the constraints (3.10), (3.9), (2.10), and (3.5). The conditions for local minima are found to be as in section 2. However, the constraints (3.5) together with the fact that $\kappa_{p+m-1}<\vec{\lambda}_{p+m}$ relegate the vectors $w_{p+1}, \ldots, w_{p+m-1}$ to the set $S_{2}$ orthogonal to $\tilde{u}_{p}$. Furthermore, the conditions (3.5) eliminate the values $i=p+1, \ldots, p+m-1$ from the $i_{\sigma}$.

Thus we find the following theorem.

Theorem 2. Let $\kappa_{1} \leq \kappa_{2} \leq \ldots . \leq \kappa_{M}$ be the Rayleigh-Ritz upper bounds for the first $M$ eigenvalues $\lambda_{1} \leq \lambda_{2} \leq \ldots$ of an hermitian operator $A$. Let $\bar{\lambda}_{1} \leq \ldots \leq \bar{\lambda}_{N}$ be lower bounds for the first $N \geq M$ eigenvalues. For a certain $p$ and $m$ let 


$$
\begin{gathered}
\kappa_{p-1}<\bar{\lambda}_{p}, \\
\kappa_{p+m-1}<\bar{\lambda}_{p+m}
\end{gathered}
$$

Define the numbers $i_{1}, i_{2}, \ldots, i_{L+1}, \beta_{1}, \ldots, \beta_{L}$ by (2.40) with $\bar{\lambda}_{p+1}, \ldots, \bar{\lambda}_{p+m-1}$ eliminated from the set of $\bar{\lambda}_{i}$, and $\kappa_{p+1}, \ldots, \kappa_{p+m-1}$ eliminated from the set of $\kappa_{\alpha}$.

Let $w_{p}$ be the Rayleigh-Ritz eigenvector that gives the bound $\kappa_{p}$. Then there exists a linear combination of unit length $\tilde{u}_{p}$ of the eigenvectors $u_{p}, \ldots ., u_{p+m-1}$ of $A$ such that

$$
\begin{array}{r}
{\left[1-\frac{1}{2}\left(w_{p}-\tilde{u}_{p}, w_{p}-\tilde{u}_{p}\right)\right]^{2} \geq\left\{1-\frac{\kappa_{p}-\bar{\lambda}_{p}}{\bar{\lambda}_{i_{L+1}}-\bar{\lambda}_{p}}\right\}} \\
\prod_{\substack{\nu=1 \\
\beta_{\nu} \neq p}}^{L}\left\{1-\frac{\left(\kappa_{p}-\bar{\lambda}_{p}\right)\left(\kappa_{\beta_{\nu}}-\bar{\lambda}_{i_{\nu}}\right)}{\left(\kappa_{p}-\kappa_{\beta_{\nu}}\right)\left(\bar{\lambda}_{p}-\bar{\lambda}_{i_{\nu}}\right)}\right\}
\end{array}
$$

As in section 2 we obtain a simpler but a weaker inequality by putting $\bar{\lambda}_{i}=\bar{\lambda}_{p+m}$ for $i>p+m$ and $\bar{\lambda}_{i}=\bar{\lambda}_{1}$ for $i<p$ when $p>1$.

This leads to

$$
\begin{array}{r}
{\left[1-\frac{1}{2}\left(w_{p}-\tilde{u}_{p}, w_{p}-\tilde{u}_{p}\right)\right]^{2} \geq\left\{1-\frac{\kappa_{p}-\bar{\lambda}_{p}}{\bar{\lambda}_{p+m}-\bar{\lambda}_{p}}\right\}} \\
\left\{1-\frac{\left(\kappa_{p}-\bar{\lambda}_{p}\right)\left(\kappa_{p-1}-\bar{\lambda}_{1}\right)}{\left(\kappa_{p}-\kappa_{p-1}\right)\left(\bar{\lambda}_{p}-\bar{\lambda}_{1}\right)}\right\} .
\end{array}
$$

For $p=1$ we only have to put $\bar{\lambda}_{i}=\bar{\lambda}_{p+m}$ for $i>p+m$ to obtain

$$
\left[1-\frac{1}{2}\left(w_{1}-\tilde{u}_{1}, w_{1}-\tilde{u}_{1}\right)\right]^{2} \geq 1-\bar{\lambda}_{m+1}-\bar{\lambda}_{1}-\bar{\lambda}_{1} .
$$

By the same reasoning we can show that there is a linear combination $\tilde{u}_{p+q}$ of $u_{p}, \ldots, u_{p+m-1}$ that approximates $w_{p+q}$ with $0<q<m$. We eliminate $\kappa_{p}, \ldots, \kappa_{p+m-1}$ except for $\kappa_{p+q}$ from the $\kappa_{\alpha}$ and $\bar{\lambda}_{p+1}, \ldots, \bar{\lambda}_{p+m-1}$ from the $\bar{\lambda}_{i}$ in forming the sets $\beta_{v}$ and $i_{\sigma}$. Then we obtain the inequalities (3.12) and (3.13) with $w_{p}$ replaced by $w_{p+q}, \tilde{u}_{p}$ by $\tilde{u}_{p+q}$, and $\kappa_{p}$ by $\kappa_{p+q}$.

Example. We consider the vibrations of a uniform beam which is free at its ends and which lies on an elastic foundation with small linearly varying elastic constant. It satisfies the differential equation

$$
u^{\mathrm{IV}}+\epsilon x u=\lambda u \quad 0<x<1
$$

with the end conditions

$$
u^{\prime \prime}(0)=u^{\prime \prime \prime}(0)=u^{\prime \prime}(1)=u^{\prime \prime \prime}(1)=0
$$

The constant $\epsilon$ is positive and small. We introduce the scalar product

$$
(u, v)=\int_{0}^{1} u v d x
$$

and define the symmetric operator $A$ by the bilinear form

$$
(A u, v)=\int_{0}^{1}\left[u^{\prime \prime} v^{\prime \prime}+\epsilon u v\right] d x
$$

If the eigenvalues $\lambda_{1} \leq \lambda_{2} \leq \ldots$ of $A$ are defined as the successive minima of the Rayleigh quotient $(A v, v) /(v, v)$, they coincide with those of the problem $(3.15),(3.16)$.

We are concerned with the two lowest eigenvalues. For $\epsilon=0$ they both vanish. Corresponding eigenfunctions are 1 and $6^{-1 / 2}(1-2 x)$. We use these as trial functions in the Rayleigh-Ritz method. We obtain

$$
\begin{aligned}
& \kappa_{1}=\frac{1}{2}\left(1-6^{-1 / 2}\right) \epsilon, \\
& \kappa_{2}=\frac{1}{2}\left(1+6^{-1 / 2}\right) \epsilon,
\end{aligned}
$$

with the corresponding vectors

$$
\begin{aligned}
& w_{1}=2^{-1 / 2}\left[1-6^{-1 / 2}(1-2 x)\right] \\
& w_{2}=2^{-1 / 2}\left[1+6^{-1 / 2}(1-2 x)\right]
\end{aligned}
$$

To obtain lower bounds $\bar{\lambda}_{i}$ we note that $(A v, v)$ lis greater for $\epsilon>0$ than for $\epsilon=0$. Thus, the $\lambda_{i}$ are bounded below by the eigenvalues $\lambda_{i}$ of the problem (3.15), (3.16) with $\epsilon=0$. These can be found explicitly. We find

$$
\begin{aligned}
& \bar{\lambda}_{1}=\bar{\lambda}_{2}=0 \\
& \bar{\lambda}_{3}=500.462 .
\end{aligned}
$$

Condition (2.39) is violated so that we cannot say how well $w_{1}$ approximates $u_{1}$ without improving our bounds. However, we can use theorem 2 to state that there are linear combinations $\tilde{u}_{1}$ and $\tilde{u}_{2}$ of $u_{1}$ and $u_{2}$ such that

$$
\begin{aligned}
& \int_{0}^{1}\left(w_{1}-\tilde{u}_{1}\right)^{2} d x \leq 2\left[1-\{1-0.0005912 \epsilon\}^{1 / 2}\right], \\
& \int_{0}^{1}\left(w_{2}-\tilde{u}_{2}\right)^{2} d x \leq 2\left[1-\{1-0.0014070 \epsilon\}^{1 / 2}\right] .
\end{aligned}
$$

Thus we have shown that $w_{1}$ and $w_{2}$ approximate linear combinations of $u_{1}$ and $u_{2}$ in the mean square sense.

When, as in this example, $A$ is unbounded, it is often more desirable to have a bound for the deviation $\left(A\left(w_{p}-\tilde{u}_{p}\right), w_{p}-\tilde{u}_{p}\right)$ rather than $\left(w_{p}-\tilde{u}_{p}, w_{p}-\tilde{u}_{p}\right)$ In order to obtain such a bound we note that the quadratic form $(A v, v)+c(v, v)$ is positive definite for $c>-\bar{\lambda}_{1}$. 
Hence we can define a new scalar product

$$
[u, v]=(A u, v)+c(u, v) .
$$

We now define the operator $\hat{A}$ by

$$
[\hat{A} u, v]=-(u, v) \text {. }
$$

Then the eigenvectors of $\hat{A}$ are multiples of those of $A$, and its eigenvalues are $-\left(\lambda_{i}+c\right)^{-1}$. Applying theorems 1 and 2 to $A$ and expressing the results in terms of $\hat{A}$, we find the following.

Theorem 3. Under the hypotheses of theorem 2 there exists for any constant $c>-\bar{\lambda}_{1}$ a linear combination $\tilde{\tilde{u}}$ of $u_{p}, \ldots, u_{p+m-1}$ such that

and

$$
(A \tilde{\tilde{u}}, \tilde{\tilde{u}})+c(\tilde{\tilde{u}}, \tilde{\tilde{u}})=\kappa_{p}+c
$$

$$
\begin{aligned}
{\left[\kappa_{p}+c+\right.} & \left.\frac{1}{2}\left\{\left(A w_{p}-A \tilde{\tilde{u}}, w_{p}-\tilde{\tilde{u}}\right)+c\left(w_{p}-\tilde{\tilde{u}}, w_{p}-\tilde{\tilde{u}}\right)\right\}\right]^{2} \\
\geq\left(\kappa_{p}+c\right)\left(\bar{\lambda}_{p}+c\right) & \left\{1-\frac{\kappa_{p}-\bar{\lambda}_{p}}{\bar{\lambda}_{i_{L}+1}-\bar{\lambda}_{p}}\right\} \prod_{\substack{\nu=1 \\
\beta_{\nu} \neq \neq_{p}}}^{L} \\
& \left\{1-\frac{\left(\kappa_{p}-\bar{\lambda}_{p}\right)\left(\kappa_{\beta_{\nu}}-\bar{\lambda}_{i_{\nu}}\right)}{\left(\kappa_{p}-\kappa_{\beta_{\nu}}\right)\left(\bar{\lambda}_{p}-\bar{\lambda}_{i_{\nu}}\right)}\right\}
\end{aligned}
$$

When the multiplicity $m=1, \tilde{\tilde{u}}$ is a multiple of $u_{p}$ and we have the analog of theorem 1.

Applying theorem 3 to the example (3.15), (3.16), we obtain the inequality

$$
\begin{aligned}
& \left.\int_{0}^{\prime} \tilde{u}^{\prime \prime 2}+(c+\epsilon x)\left(w_{1}-\tilde{\tilde{u}}\right)^{2}\right] d x \leq \\
& \quad 2\left[c+1.704 \epsilon-c^{1 / 2}\{c+1.704 \epsilon\}^{1 / 2}\{1-0.0005912 \epsilon\}^{1 / 2}\right]
\end{aligned}
$$

for any $c \geq 0$. (The function $\tilde{\tilde{u}}$ depends upon $c$, however.)

\section{Pointwise Bounds for Eigenfunctions}

When $A$ is a differential operator, theorems 1 and 3 give bounds for the mean square deviation of the approximate eigenfunction $w_{p}$ from the exact eigenfunction $u_{p}$. It is often of interest to determine the value of $u_{p}$ at a particular point.

In certain cases a pointwise bound for the deviation $\left|w_{p}-\tilde{\tilde{u}}\right|$ at a point comes directly from the bound (3.26) of theorem 3. For example, we show that for any $0 \leq \xi \leq 1$

$$
\left|w_{1}(\xi)-\tilde{\tilde{u}}(\xi)\right|^{2} \leq G(\xi, \xi) \int_{0}^{1}\left[\left(w_{1}-\tilde{\tilde{u}}\right)^{\prime \prime 2}+c\left(w_{1}-\tilde{\tilde{u}}\right)^{2}\right] d x
$$

where

$$
G(\xi, \xi)=\frac{1}{8} b^{-3}\left[\sinh ^{2} b-\sin ^{2} b\right]^{-1}
$$

$\times[\sinh b \cosh b-\sin b \cos b$

$+2 \sinh b \cosh b(1-2 \xi)-2 \sin b \cos b(1-2 \xi)$

$+(\sinh b \cos b+\sin b \cosh b) \sin b(1$

$-2 \xi) \sinh b(1-2 \xi)+(\sinh b \cos b$

$-\sin b \cosh b) \cos b(1-2 \xi) \cosh b(1-2 \xi)]$

$b=\left(\frac{1}{4} c\right)^{1 / 4}$

(See, for example, [8]). Thus, (3.27) gives a bound for $\left|w_{1}(\xi)-\tilde{\tilde{u}}(\xi)\right|$.

In the case of partial differential operators such a bound may or may not exist. If it exists, it is difficult to find.

However, one can use the following adaptation of the method of Diaz and Greenberg $[7,9]$ ) cf. $[15,18])$. For the sake of simplicity we present it only for the case of a special second order operator in two dimensions.

Consider the eigenvalue problem

$$
-\Delta u+r(x, y) u=\lambda q(x, y) u
$$

on a two-dimensional domain $D$ with smooth boundary $C$. Here $\Delta$ is the usual Laplace operator. The function $q$ is positive and $r$ is non-negative, and both are continuous in the closure of $D$. The boundary $C$ consist of two parts $C_{1}$ and $C_{2}$, and we have boundary conditions

$$
\begin{aligned}
& u=0 \quad \text { on } \quad C_{1} \\
& \frac{\partial u}{\partial n}+k(x, y) u=0 \quad \text { on } \quad C_{2}, \quad k \geq 0 .
\end{aligned}
$$

We define the scalar product

$$
(u, v)=\iint_{D} q u v d x d y
$$

on the linear vector space of functions which are piecewise continuously differentiable in $D$ and vanish on $C_{1}$.

Let $u_{p}$ be the normalized eigenfunction corresponding to the eigenvalue $\lambda_{p}$, and let $w_{p}$ be the function corresponding to a Rayleigh-Ritz upper bound $\kappa_{p}$ for $\lambda_{p}$. Theorem 1 gives a bound for the deviation in norm $\left(w_{p}-u_{p}, w_{p}-u_{p}\right)$.

We wish to approximate the value of $u_{p}$ at an interior point of $D$, which we choose as the origin of our coordinate system. We use the fact that $u_{p}$ satisfies the differential equation (4.3) with $\lambda=\lambda_{p}$ and the boundary conditions (4.4). Let $\Gamma(x, y)$ be a parametrix for the differential equation (4.3) satisfying (4.4). That is,

$$
\Gamma(x, y)=-(4 \pi)^{-1} \log \left(x^{2}+y^{2}\right)+\phi(x, y),
$$

where $\phi$ is any twice continuously differentiable function such that 


$$
\begin{aligned}
\Gamma=0 & \text { on } \quad C_{1} \\
\frac{\partial \Gamma}{\partial n}+k \Gamma=0 & \text { on } \quad C_{2}
\end{aligned}
$$

Multiplying (4.3) by $\Gamma$ and integrating by parts, we find that

$$
u_{p}(0,0)=\iint_{D} u_{p}\left[-\Delta \phi+\left(r-\lambda_{p} q\right) \Gamma\right] d x d y .
$$

Replacing $u_{p}$ by $w_{p}$ and $\lambda_{p}$ by $\kappa_{p}$ on the right, we obtain the value

$$
w_{p}^{*}(0,0)=\iint_{D} w_{p}\left[-\Delta \phi+\left(r-\kappa_{p} q\right) \Gamma\right] d x d y
$$

which can be computed by quadratures. Using Schwarz's inequality, the normalization of $u_{p}$, and the triangle inequality, we find

$$
\begin{gathered}
\left|w_{p}^{*}(0,0)-u_{p}(0,0)\right| \leq\left(\kappa_{p}-\lambda_{p}\right)(\Gamma, \Gamma)^{1 / 2} \\
+\left(w_{p}-u_{p}, w_{p}-u_{p}\right)^{1 / 2} \\
\left\{\iint_{D} q^{-1}\left[-\Delta \phi+\left(r-\kappa_{p} q\right) \Gamma\right]^{2} d x d y\right\}^{1 / 2}
\end{gathered}
$$

Thus, the bound (2.41) for $\left(w_{p}-u_{p}, w_{p}-u_{p}\right)$ together with the bound $\kappa_{p}-\bar{\lambda}_{p}$ for $\kappa_{p}-\lambda_{p}$ provides explicit upper and lower bounds for $u_{p}(0,0)$. These bounds lie close together if the error bounds (2.41) and $\kappa_{p}-\bar{\lambda}_{p}$ are small.

The same method applies to the function $\tilde{u}_{p}$ of theorem 2. If

$$
\tilde{u}_{p}=\sum_{p}^{p+m-1} a^{i} u_{i}
$$

we find from (4.8) that

$$
\tilde{u}_{p}(0,0)=\sum a^{i} \iint_{D} u_{i}\left[-\Delta \phi+\left(r-\lambda_{i} q\right) \Gamma\right] d x d y .
$$

Hence if we again define the approximate value $w_{p}^{*}$ by (4.9), we obtain

$$
\begin{gathered}
\left|w_{p}^{*}(0,0)-\tilde{u}_{p}(\mathbf{0}, 0)\right| \leq \max \left\{\kappa_{p}-\bar{\lambda}_{p}, \kappa_{p+m-1}-\kappa_{p}\right\} \\
(\Gamma, \Gamma)^{1 / 2}+\left(w_{p}-\tilde{u}_{p}, w_{p}-\tilde{u}_{p}\right)^{1 / 2} \\
\left\{\iint_{D} q^{-1}\left[-\Delta \phi+\left(r-\kappa_{p} q\right) \Gamma\right]^{2} d x d y\right\}^{1 / 2} \cdot
\end{gathered}
$$

This inequality together with theorem 2 gives upper and lower bounds for $\tilde{u}_{p}(0,0)$.

\section{References}

[1] N. Aronszajn, The Rayleigh-Ritz and the Weinstein methods for approximation of eigenvalues, Oklahoma A and M College Tech. Reports 1-4 (1949-1950).

[2] N. Bazley, Lower bounds for eigenvalues with applications to the helium atom, Proc. Nat. Acad. Sci. 45 (1959).

[3] G. Bertram, Fehlerabschätzung für das Ritz-Galerkinsche Verfahren bei Eigenwertproblemen, Z.A.M.M. 37, 191201 (1957).

[4] W. Börsch-Supan, Obere Schranken für den gröszten Eigenwert eines vollstetigen selbstadjungierten Operators, Math. Ann. 134, 453-457 (1958).

[5] L. Collatz, Eigenwertprobleme und ihre numerische Behandlung (Chelsea Press, New York, N.Y., 1948).

[6] J. B. Diaz, Upper and lower bounds for eigenvalues, Proc Eighth Symp. on Appl. Math. of the A.M.S., pp. 53-78 (Calculus of Variations and its Applications, 1956).

[7] J. B. Diaz and H. J. Greenberg, Upper and lower bounds for the solution of the first biharmonic boundary value problem, J. Math. Phys. 27, 193-201 (1948).

[8] M. Golomb and H. F. Weinberger, Optimal approximations and error bounds, On Numerical Approximation (Univ. of Wisconsin Press, Madison, Wis., 1959).

[9] H. J. Greenberg, The determination of upper and lower bounds for the solution of the Dirichlet problem, J. Math. Phys. 27, 161-182 (1948).

[10] A. Hammerstein, Eine Restabschätzung für das Ritzsche Verfahren bei gewissen Variationsproblemen mit Nebenbedingungen, Sitzungsberichte d. Berliner Math. Ges. 26, 171-177 (1927).

[11] F. Koehler, Estimates for eigenvalues of infinite matrices, Pac. J. Math. 7, 1391-1404 (1957).

[12] N. Kryloff, Les méthodes de solution approchée des problèmes de la physique mathématique, Mém. des Sci. Math. 49 (1931).

[13] N. J. Lehmann, Beiträge zur numerischen Lösung linearer Eigenwertprobleme, Z.A.M.M. 29, 342-356 (1949); 30, $1-16$ (1950).

[14] H. J. Maehly, Ein neues Variationsverfahren zur genäherten Berechnung der Eigenwerte hermitescher Operatoren, Helv. Phys. Acta \$5, 547-568 (1952).

[15] C. G. Maple, The Dirichlet problem bounds at a point for the solution and its derivatives, Quart. Appl. Math. 8, 213-228 (1950).

[16] M. Parodi, Sur quelques proprietes des valeurs charactéristiques des matrices carrés, Mém. Sci. Math. 118 (1952).

[17] J. F. Steffensen, Interpolation (Chelsea, New York, 1950).

[18] J. L. Synge, Pointwise bounds for the solutions of certain boundary value problems. Proc. Roy. Soc. [A] 208, $170-175$ (1951).

[19] G. Temple and W. G. Bickley, Rayleigh's principle and its applications to engineering, London (1933).

[20] E. Trefftz, Über Fehlerabschätzung bei berechnung von Eigenwerten. Math. Ann. 108, 595-604 (1933).

[21] H. F. Weinberger, Upper and Lower Bounds for Eigenvalues by Finite Difference Methods. Com. Pure Appl. Math. 9, 613-623 (1956), (Also, Proc. Conf. on Partial Diff. Eq., Berkeley, Calif., 1956).

[22] H. F. Weinberger, Lower bounds for higher eigenvalues by finite difference methods. Pacific J. Math. 8, 339-368 (1958).

[23] H. F. Weinberger, A theory of lower bounds for eigenvalues. Inst. for Fluid Dyn. and Applied Math., Univ. of Md., Technical Note BN 183 (1959).

[24] A. Weinstein, Etude des spectres des équations aux dérivées partielles de la théorie des plaques élastiques. Mém. des Sci. Math. 88 (1937).

[25] A. Weinstein, Variational methods for the approximation and exact computation of eigenvalues. NBS Applied Math. Series 29 p. 83 (1953).

[26] H. Wielandt, Einschliessung von Eigenwerten nach dem Abschnittsverfahren. Archiv. d. Math. 5, 108-114 (1954).

(Paper 64B4-37) 